\title{
Strongly correlated photons on a chip
}

\author{
Andreas Reinhard, ${ }^{1, \text { * }}$ Thomas Volz, ${ }^{1}$, ${ }^{*}$ Martin Winger, ${ }^{1}$ Antonio \\ Badolato, ${ }^{2}$ Kevin J. Hennessy, ${ }^{1}$ Evelyn L. Hu, ${ }^{3}$ and Ataç Imamoğlu ${ }^{1}$ \\ ${ }^{1}$ Institute of Quantum Electronics, ETH Zurich, 8093 Zurich, Switzerland \\ ${ }^{2}$ Department of Physics and Astronomy, University of Rochester, Rochester, NY 14627, USA \\ ${ }^{3}$ School of Engineering and Applied Physics, Harvard University, Cambridge, Massachusetts 02138, USA
}

(Dated: November 3, 2018)

Optical non-linearities at the single-photon level are key ingredients for future photonic quantum technologies [1]. Prime candidates for the realization of strong photon-photon interactions necessary for implementing quantum information processing tasks [2] as well as for studying strongly correlated photons [3, 4] in an integrated photonic device setting are quantum dots embedded in photonic crystal nanocavities. Here, we report strong quantum correlations between photons on picosecond timescales. We observe (a) photon antibunching upon resonant excitation of the lowest-energy polariton state, proving that the first cavity photon blocks the subsequent injection events, and (b) photon bunching when the laser field is in two-photon resonance with the polariton eigenstates of the second Jaynes-Cummings manifold, demonstrating that two photons at this color are more likely to be injected into the cavity jointly, than they would otherwise. Together, these results demonstrate unprecedented strong single-photon non-linearities, paving the way for realizing a single-photon transistor [5] or a quantum optical Josephson interferometer [6].

Cavity quantum electrodynamics (cQED) studies the quantum limit of light-matter interaction where a single two-level quantum emitter is coupled to a single cavity mode 2. In the strong coupling regime of cavityQED where the coherent interaction strength between the emitter and the cavity mode exceeds the dissipative rates, the elementary excitations (polaritons) have an anharmonic spectrum (Fig. 1a). As a consequence, this system embodies the ultimate non-linear optical device enabling the observation of photon-photon interactions at the single-photon level [7]. Various implementations of cavity-QED systems have been reported with atoms in high finesse cavities [8], with quantum dots (QDs) in different types of monolithic cavities [9-12] and in the microwave domain [13 15]. Recent experiments in the optical domain using a single atom coupled to FabryPerot [16] or toroidal cavities [17] have demonstrated the

\footnotetext{
${ }^{*}$ These authors contributed equally to this work.
}

a

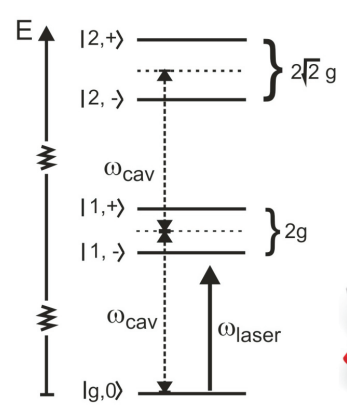

b
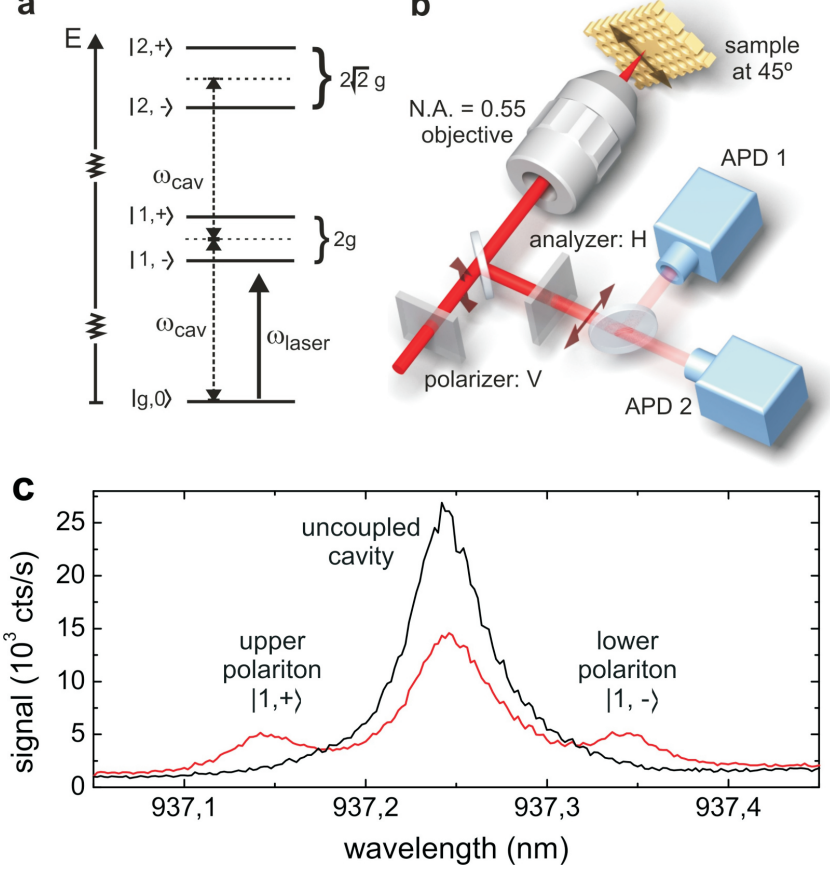

FIG. 1: Resonant scattering spectroscopy of a QD strongly coupled to a PC cavity. a) Non-linear JaynesCummings level scheme up to the second manifold. b) Sketch of the experimental setup with crossed-polarized laser suppression. c) On-resonance cw scattering spectrum for a probe power of $1 \mathrm{nW}$. The black trace was recorded without the additional re-pump laser. With the re-pump switched on, the resonant signal from the polaritons is restored (red trace).

photon blockade effect by observing photon antibunching in correlation measurements. Photon bunching upon two-photon excitation of the second Jaynes-Cummings manifold has been observed for a single atom cavity-QED system 18. In the solid-state, early results in quantum dot (QD) cavity-QED systems indicating optical nonlinearities have been reported [19, 20].

Here, we show that a single QD deterministically coupled to a photonic crystal (PC) defect cavity exhibits pronounced photon antibunching as witnessed by a reduction in two-photon scattering events from the strongly coupled polariton mode by more than $40 \%$ upon resonant excitation - a clear indication of photon blockade. By tuning either the laser or the cavity mode frequency to ensure resonant two-photon excitation of the higher 
polariton states [21], we observe that the photon emission is strongly bunched with an increase in simultaneous two-photon scattering events by $50 \%$. Hence, we demonstrate for the first time direct access to the second manifold of the Jaynes-Cummings ladder in an integrated solid-state photonic device.

By positioning an InAs/GaAs QD at an electric-field antinode of a PC defect cavity in L3 geometry [12, we achieve a coherent coupling constant $g$ of $141 \mu \mathrm{eV}$. The cavity has a quality factor $\mathrm{Q}$ of about 25000 , corresponding to a cavity photon decay rate $\kappa$ of $53 \mu \mathrm{eV}$. In order to tune the cavity frequency, we employ a thin-film deposition technique using nitrogen gas injection [22. A crossed-polarization technique ensures efficient suppression of the excitation-laser light which is back-reflected from the sample surface [23, 24]; the setup is sketched in Fig. 1b.

A large majority of QDs [25], as well as most other solid-state based emitters such as NV centers [26, exhibit the phenomenon of blinking, induced by spontaneous or induced change of the internal state of the QD, and leading to an intermittent optical response. Experimentally, we find that the fractional amount of time our system spends in the neutral QD ground state strongly decreases with increasing resonant probe power, and is vanishingly small above $1 \mathrm{nW}$ (see Supplementary Material). Incident laser photons thus simply scatter off the uncoupled cavity, resulting in a spectrum as shown in Fig. 1c (black trace). In order to overcome this problem, we use an off-resonant laser just below the edge of the wetting layer to re-pump the QD into its neutral ground state. By alternating re-pump and probe intervals, we partly recover the polariton spectrum shown in Fig 1c (red trace).

We carry out correlation measurements using resonant pulses at a duration of $T_{\text {pulse }} \approx 72$ ps (FWHM), yielding a ratio between pulse length and polariton lifetime of $\frac{\kappa}{2} \cdot T_{\text {pulse }} \approx 2.9$. Fig. 2 displays correlation histograms for different laser/cavity detunings. The respective detunings are indicated in the resonant scattering spectrum shown in Fig 2a. We define the correlation function for pulsed excitation at zero time delay $g_{\text {pulsed }}^{(2)}(0)$ as the area of the central peak divided by the average area of peaks at other times (see Supplementary Material). First, we choose the frequency of the pulsed laser to match the upper polariton transition and obtain significant antibunching with $g_{\text {pulsed }}^{(2)}(0)=0.75 \pm 0.06$ (Fig. 2c). For comparison, we turn off the re-pump laser and verify that photons scattered from the uncoupled cavity mode have Poissonian statistics (Fig. 2b). As a cross-check, we also confirmed that the scattered photons from a resonantly driven cavity mode that is far detuned from all QD transitions exhibit Poissonian statistics. These experiments jointly demonstrate that the applied re-pump laser can be used to switch on the single-photon non-linearity and
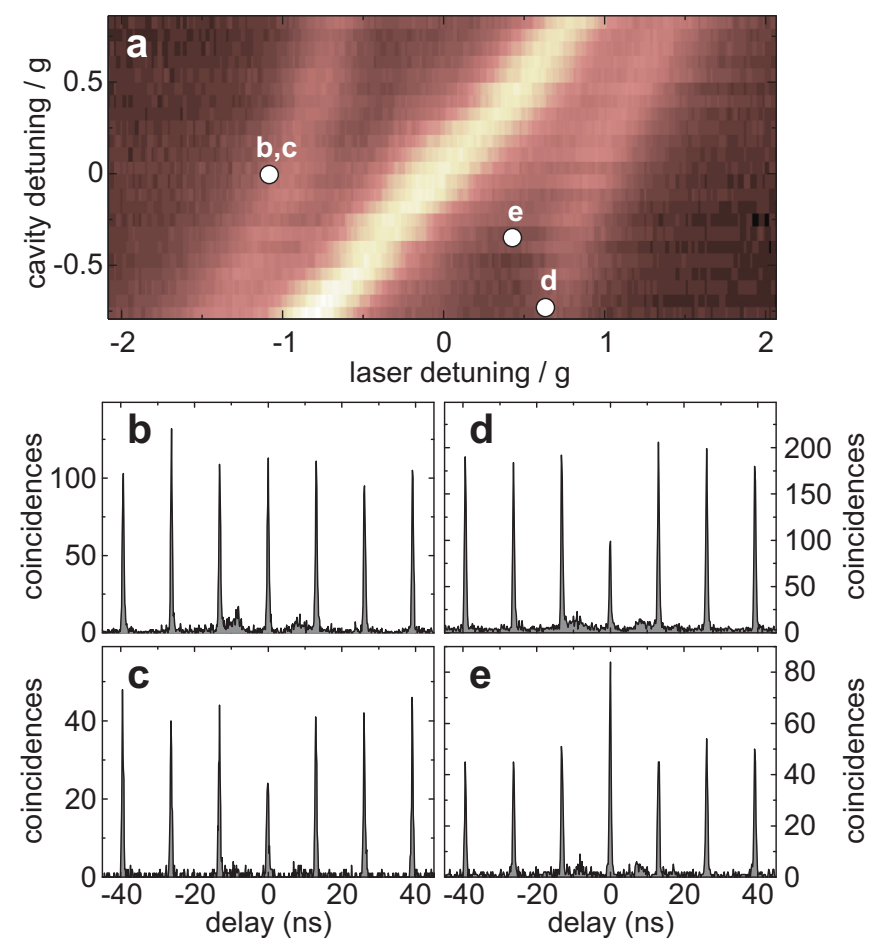

FIG. 2: From Poissonian light to antibunched and bunched photon streams. a) Resonant scattering spectra for different cavity detunings close to the neutral exciton resonance. The circles indicate the detunings for which the correlation histograms in $\mathrm{b}-\mathrm{e}$ were taken. The vertical axes in the histograms specify the photon coincidences per timebin of 192 ps. b) Autocorrelation histogram recorded on exact cavity-exciton resonance without re-pump laser for a detuning corresponding to the energy of the upper polariton. c) Autocorrelation with the same detunings, but with a re-pump laser applied. The scattered photons in this case exhibit photon antibunching. d) Autocorrelation on the lower polariton for a blue detuning of the cavity of $0.68 \mathrm{~g}$, where the longer polariton lifetime ensures stronger photon antibunching. e) Pronounced photon-bunching on the lower two-photon resonance. Note that the small features at $\approx \pm 10 \mathrm{~ns}$ in $\mathrm{b}-\mathrm{e}$ originate from cross-talk between the APDs due to secondary photon-emission events.

to control the statistics of the scattered photons. We emphasize that the degree of photon antibunching observed in the experiment is limited by the finite excitation pulse length. Accordingly, a higher degree of antibunching $\left(g_{\text {pulsed }}^{(2)}(0)=0.57 \pm 0.02\right)$ is observed when the cavity is off-resonant from the exciton, as shown in Fig. 2d for the lower polariton in the case of blue-detuning of the cavity mode; here the polariton state lifetime is prolonged, making multiple photon absorption events within a single pulse less likely. Experimentally, we do not observe a difference between correlations recorded on the upper and lower polariton if the respective laser and cavity detunings from the exciton change their sign.

When tuning the laser photon energy to half the 
energy of the lower polariton eigenstate of the second Jaynes-Cummings manifold, we observe photonbunching. Fig. 2e displays a corresponding correlation histogram with $g_{\text {pulsed }}^{(2)}(0)=1.5 \pm 0.1$. The bunching has its origin in a two-photon transition from the ground state to the second manifold of the Jaynes-Cummings ladder. We emphasize that the non-vanishing correlations at zero time delay in the case of photon blockade, and the moderate bunching-feature on the two-photon transition have their origin mostly in the particular implementation of the measurement using pulsed laser excitation and slow single-photon detectors; in order to confirm that and to explain the principal experimental features, we carried out numerical simulations of $g_{\text {pulsed }}^{(2)}(0)$ using a Monte Carlo wave function (MCWF) approach (see Supplementary Material). Here, we directly accounted for the pulsed laser excitation, the non-zero laser background due to imperfect extinction of the laser reflection and the uncoupled cavity resonance due to the blinking of the dot. Fig. 3a displays the resulting autocorrelation function $g_{\text {pulsed }}^{(2)}(0)$ at zero time delay for varying cavity and laser detunings.

In addition to the bunching features originating from two-photon resonances, we would normally expect strong bunching when the external laser that drives the cavity mode is resonant with the bare exciton [18, as can be seen in Fig. 3a (dotted vertical line). Unlike in previous experiments [19], this bunching-feature is absent in our experiments as well as in the simulations when the cavity frequency is resonant with the bare QD exciton, since for this detuning we predominantly detect photons from instances when the QD remains charged and is thus offresonant. Conversely, our experiments demonstrate for the first time that a coupled QD-cavity device realizes the anharmonic Jaynes-Cummings model [30]. In the simulations, we assumed a pure QD dephasing rate of $\hbar \gamma_{\text {deph }}=13 \mu \mathrm{eV}$ in addition to the cavity dissipation rate $\kappa$, consistent with the polariton linewidths observed in the experiment. This line broadening might stem from charge fluctuations in the QD environment, partially induced by the re-pump laser, and from phonon-induced dephasing.

In Fig. $3 \mathrm{~b}$ and $3 \mathrm{c}$ we probe the upper and lower polariton branches for constant cavity detunings of $(-0.33 \pm 0.04) g$ and $(1.04 \pm 0.03) g$, respectively, by tuning the laser wavelength. The continuous change in photon correlations from strong antibunching to bunching maps out the (anharmonic) spectrum of the JaynesCummings ladder. In addition, we perform correlation measurements for a varying cavity-exciton detuning at a constant laser-exciton detuning of $(0.94 \pm 0.05) \mathrm{g}$, demonstrating that the nature of strong photon correlations can be tuned by changing either the laser or the cavity mode frequency. The agreement between experimental values and theoretical expectation is very good
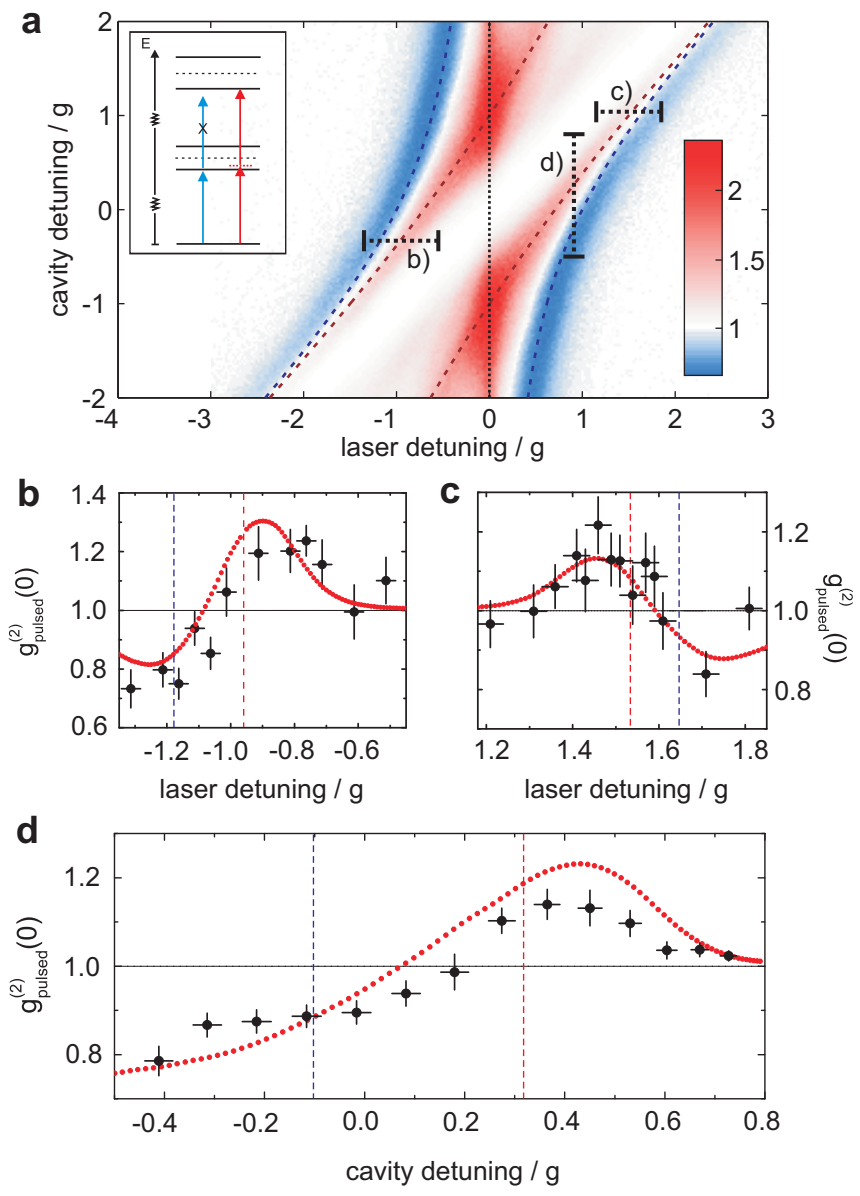

FIG. 3: Calculated and measured autocorrelation functions $g_{\text {pulsed }}^{(2)}(0)$ for different cavity and laser detunings. a) Results of a MCWF simulation close to the neutral exciton-cavity resonance using experimental parameters determined from linear resonant light scattering measurements. The blue regions correspond to detunings with sub-Poissonian statistics, whereas the red regions indicate photon-bunching. Blue and red dashed lines denote the expected positions of the polariton frequencies and the two-photon resonances, respectively. The enhanced bunching feature along the dotted vertical line at zero detuning is a consequence of an interference effect, ensuring small occupancy of the cavity mode. The bold dotted lines indicate the parameter ranges of the three different data traces displayed in $\mathrm{b}-\mathrm{d}$. There, the red dotted traces are the theoretical expectations from the MCWF simulations.

in all cases; we emphasize in this regard that the theoretical curves provide a prediction based on independently determined parameters. The dashed vertical lines in Fig. 3b-d correspond to the polariton energy (blue) and the energy of the two-photon transition to the second manifold (red), indicating the origin of both the antibunching and the bunching features.

An obvious extension of the results presented here includes non-linear optics experiments at the single-photon level, e.g. the realization of a single-photon transistor [5. Furthermore, our results elevate this system to an 
ultimate optical non-linear building block for more complex structures investigating strong photon correlations in non-equilibrium settings, such as an optical Josephson interferometer [6] or coupled arrays of non-linear cavities [3, 4, 27. In connection with recent progress on the fabrication of site-controlled QDs within arrays of photonic crystal cavities [28] and the ability to tune QD transitions by up to $25 \mathrm{meV}$ in p-i-n structures 29], the present work demonstrates the great potential of QD-cavity systems as candidates for photonic quantum simulators.

This work is supported by NCCR Quantum Photonics (NCCR QP), research instrument of the Swiss National Science Foundation (SNSF) and an ERC Advanced Investigator Grant (A.I.). The authors thank Iacopo Carusotto for helpful discussions. The authors declare that they have no competing financial interests. Correspondence and requests for materials should be addressed to T.V. and A.I. (E-mail: volz@phys.ethz.ch, imamoglu@phys.ethz.ch)

\section{Methods}

\section{Optical characterization}

Optical characterization is performed in a liquid helium flow cryostat at a temperature $T=5-7 \mathrm{~K}$. A $50 \times$ microscope objective $(\mathrm{NA}=0.55)$ is used to illuminate the defect region of the $\mathrm{PC}$ with a spot size of about $1 \mu \mathrm{m}$. Emitted photons are collected with the same objective. After cooldown, the cavity luminesces at a wavelength of around $935 \mathrm{~nm}$, as determined with photoluminescence spectroscopy. In order to tune the cavity into resonance with the neutral exciton transition, a thinfilm deposition technique using nitrogen gas injection is used. Even without active deposition of nitrogen, the cavity mode shows an intrinsic red tuning at a rate up to $0.008 \mathrm{~nm} /$ hour. For resonant scattering experiments, we excite the cavity mode with an actively power-stabilized narrow bandwidth, mode-hop-free tunable diode laser. The sample is mounted such that there is a $45^{\circ}$ angle between the polarization axes of the cavity mode and the laser. By sending the collected light through a singlemode fiber with mounted fiber-polarization controllers and a subsequent analyzer, we have precise control over the extinction of the reflected laser light. The photons scattered off the cavity are detected with an avalanche photo diode (APD) in single-photon counting mode.

\section{Off-resonant re-pump scheme}

For the off-resonant re-pumping of the system, we use an additional cw titanium-sapphire laser at a wavelength of $857.2 \mathrm{~nm}$. Using an acousto-optical modulator (AOM) we excite the $\mathrm{QD}$ with rectangular laser pulses at a repetition rate of $0.5-1 \mathrm{MHz}$. In between the re-pump pulses, we probe the system by triggering the APD readout.

\section{Autocorrelation measurements}

For photon correlation measurements, we replace the cw probe light by resonant pulses from a mode-locked titanium-sapphire laser in ps mode, with a repetition rate of $76.3 \mathrm{MHz}$. In order to filter the broadband pulses we send them through a $750 \mathrm{~mm}$ grating spectrograph and couple the diffracted light into a single-mode fiber. The transmitted pulse is approximately Gaussian with a typical width of $0.018 \mathrm{~nm}$. Using a streak camera we confirm that the resulting pulses have near-Fourier-limited durations of about $72 \mathrm{ps}$. The dependence of photon correlations on the laser frequency are obtained by rotating the grating of the spectrograph and consequently filtering out different parts of the pulse spectrum. Measurements depicting the dependence of photon correlations on the cavity resonance frequency on the other hand are carried out by exploiting an intrinsic cavity wavelength drift of about $0.006 \mathrm{~nm} / \mathrm{h}$ while keeping the laser frequency fixed. We perform autocorrelation measurements with a Hanbury-Brown and Twiss set-up, consisting of a 50:50 beam splitter and an APD at each output. Photon arrival time differences are determined with a time-toamplitude converter and plotted in a histogram.

[1] O'Brien, J. L. et al. Photonic quantum technologies. $N a$ ture Photon. 3, 687-695 (2009)

[2] Mabuchi, H. \& Doherty, A. C. Cavity quantum electrodynamics: Coherence in context. Science 298, 1372-1377 (2002).

[3] Hartmann, M. J. et al. Strongly interacting polaritons in coupled arrays of cavities. Nature Physics 2, 849-855 (2006).

[4] Greentree, A. D. et al. Quantum phase transitions of light. Nature Physics 2, 856-861 (2006).

[5] Chang, D. E. , Sørensen A. S. , Demler E. A. \& Lukin, M. A single-photon transistor using nanoscale surface plasmons. Nature Physics 3, 807-812 (2007).

[6] Gerace, D. et al. The quantum optical Josephson interferometer. Nature Physics 5, 281-284 (2009).

[7] Imamoglu, A. et al. Strongly interacting photons in a nonlinear cavity. Phys. Rev. Lett. 79, 1467-1470 (1997).

[8] Brune, M. et al. Quantum Rabi Oscillations: A Direct Test of Field Quantization in a Cavity. Phys. Rev. Lett. 76, 1800-1803 (1996).

[9] Yoshie, T. et al. Vacuum Rabi splitting with a single quantum dot in a photonic crystal nanocavity. Nature 432, 200-203 (2004).

[10] Reithmaier, J. P. et al. Strong coupling in a single quantum dot-semiconductor microcavity system. Nature 432, 197-200 (2004). 
[11] Peter, E. et al. Exciton-photon strong-coupling regime for a single quantum dot embedded in a microcavity. Phys. Rev. Lett. 95, 067401 (2005).

[12] Hennessy, K. et al. Quantum nature of a strongly coupled single quantum dot-cavity system. Nature 445, 896899 (2007).

[13] Fink, J. M. et al. Climbing the Jaynes-Cummings ladder and observing its $\sqrt{n}$ nonlinearity in a cavity QED system. Nature 454, 315-318 (2008).

[14] Deppe, F. et al. Two-photon probe of the JaynesCummings model and controlled symmetry breaking in circuit QED. Nature Physics 4, 686-691 (2008).

[15] Bishop, L. S. et al. Nonlinear response of the vacuum Rabi resonance. Nature Physics 5, 105-109 (2009).

[16] Birnbaum, K. M. et al. Photon blockade in an optical cavity with one trapped atom. Nature 436, 87-90 (2005).

[17] Dayan, B. et al. A Photon Turnstile Dynamically Regulated by One Atom. Science 319, 1062-1065 (2008).

[18] Kubanek, A. et al. Two-Photon Gateway in One-Atom Cavity Quantum Electrodynamics. Phys. Rev. Lett. 101, 203602 (2008).

[19] Faraon, A. et al. Coherent generation of non-classical light on a chip via photon-induced tunneling and blockade. Nature Physics 4, 859-863 (2008).

[20] Kasprzak, J. et al. Up on the Jaynes-Cummings ladder of a quantum-dot/microcavity system. Nature Materials 9, 304-308 (2010).

[21] Schneebeli, L. , Kira, M. \& Koch, S. W. Characterization of Strong Light-Matter Coupling in Semiconductor Quantum-Dot Microcavities via Photon-Statistics Spectroscopy. Phys. Rev. Lett. 101, 097401 (2008).

[22] Strauf, S. et al. Frequency control of photonic crystal membrane resonators by monolayer deposition. Appl. Phys. Lett. 88, 043116 (2006).

[23] Englund, D. et al. Controlling cavity reflectivity with a single quantum dot Nature 450, 857-861 (2007).

[24] Srinivasan, K. \& Painter, O. Linear and nonlinear optical spectroscopy of a strongly coupled microdisk-quantum dot system. Nature 450, 862-866 (2007).

[25] Santori, C. et al. Submicrosecond correlations in photoluminescence from InAs quantum dots. Phys. Rev. B 69, 205324 (2004).

[26] Manson, N. B. \& Harrison, J. P. Photo-ionization of the nitrogen- vacancy center in diamond. Diamond $\&$ Related Materials 14, 1705-1710 (2005).

[27] Carusotto, I. et al. Fermionized Photons in an Array of Driven Dissipative Nonlinear Cavities. Phys. Rev. Lett. 103, 033601 (2009).

[28] Gallo, P. et al. Integration of site-controlled pyramidal quantum dots and photonic crystal membrane cavities. Appl. Phys. Lett. 92, 263101 (2008).

[29] Bennett, A. J. et al. Giant Stark effect in the emission of single semiconductor quantum dots. Appl. Phys. Lett. 97, 031104 (2010).

[30] Photon antibunching can also be observed when the coupled emitter-cavity system is not in the strong coupling regime. 


\section{Supplementary Material}

\section{Laser-induced quantum dot blinking}

In resonant spectroscopy, far below saturation $p_{\text {sat }}$ of the system, the detection rate of scattered photons is expected to grow linearly with excitation power $p_{\text {exc }}$. In stark contrast, we observe highly nonlinear power dependencies at picoWatt $(\mathrm{pW})$ level, which is five orders of magnitude smaller than the $\mathrm{cw}$ saturation power $p_{\text {sat }} \approx 850 \mathrm{nW}$ (for an estimated $2 \%$ laser-cavity coupling). The polariton signal saturates at $p_{\mathrm{exc}} \approx 10 \mathrm{pW}$, whereas the uncoupled cavity resonance appears at the same power level with a super-linear power dependence, as seen in Fig. S 4a. We attribute this behaviour to laser-induced QD-blinking, which could originate from relaxation of optically excited QD states either into a dark exciton or an electron (hole) charged state. The resulting long-lived states (denoted by $|\mathrm{h}\rangle$ in Fig. S $4 \mathrm{~b}$ shift the exciton resonance off the cavity frequency, such that the cavity resonance becomes uncoupled from QD resonances. For excitation powers below $5 \mathrm{pW}$, only the polaritons appear in the spectrum. For increasing incident optical power, however, the laser-induced charge/spinpumping causes a reduction of neutral ground state occupation such that the polariton signal saturates. Above $p_{\text {exc }} \approx 100 \mathrm{pW}$, the bare cavity resonance completely dominates the spectrum.

We determine the average lifetime of these long-lived charged/dark QD states as well as the neutral ground state lifetime, by measuring the second order autocorrelation function of the uncoupled cavity and the polaritons, respectively. By extracting the lifetimes from the correlation traces, like the ones shown in Fig. S 5a, we identify the metastable charged/dark QD state lifetimes to be of several ms. In contrast, the neutral ground state lifetime is about $\approx 300 \mu \mathrm{s}$ for $p_{\text {exc }}=10 \mathrm{pW}$, with a significant reduction for higher excitation powers, as shown in Fig. S 5b 1]. This is consistent with our assumption and motivates the use of a non-resonant re-pump laser that randomly re-shuffles the internal QD state. We implement this strategy by exciting the system just below the wetting-layer resonance and observe a relaxation probability into the neutral ground state of $0.3-0.4$. Using a pump-probe scheme, we are able to partially suppress the influence of blinking and obtain a time-integrated spectrum that exhibits a three-peak feature (see Fig. 1c in the main text).

Finally, we emphasize that the blinking observed in our QD-cavity system is by no means unique and is observed by most groups working with such systems 2, 3. Depending on the particular device, however, the effect is more or less pronounced [4].

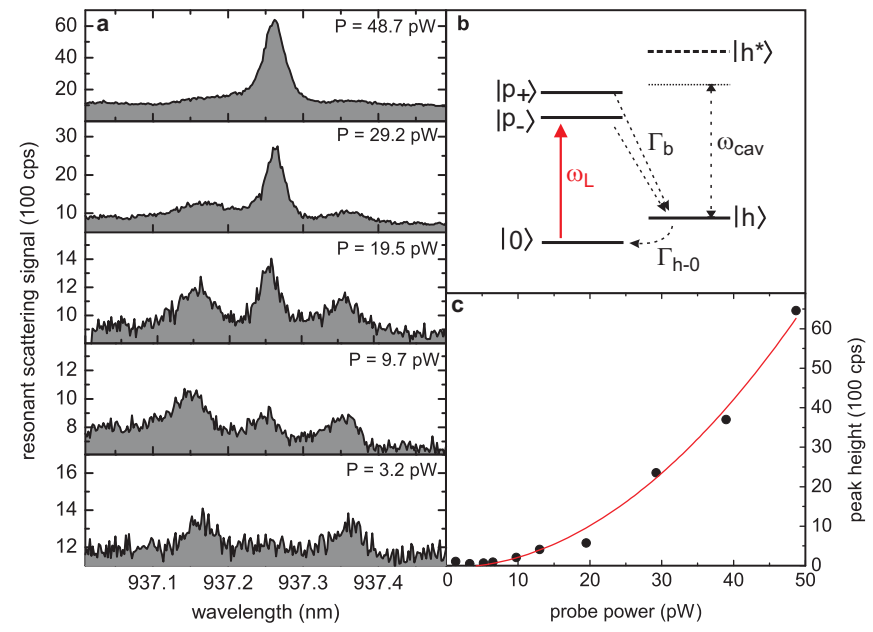

FIG. S 4: Low power spectra on cavity-exciton resonance. a) At a resonant probe power of $3.2 \mathrm{pW}$ only the polaritons are observed. For increasing excitation powers, the uncoupled cavity mode dominates the spectrum, while the polariton peaks saturate. b) Level scheme: pumping of charged or dark neutral states $|\mathrm{h}\rangle$ occur when the polaritons are excited. The recovery rate into the neutral ground state $\Gamma_{\mathrm{h}-0}$ is below $10^{3} s^{-1}$. c) Power dependent peak height of the uncoupled cavity resonance, which provides a direct measure of charged/dark state occupation.
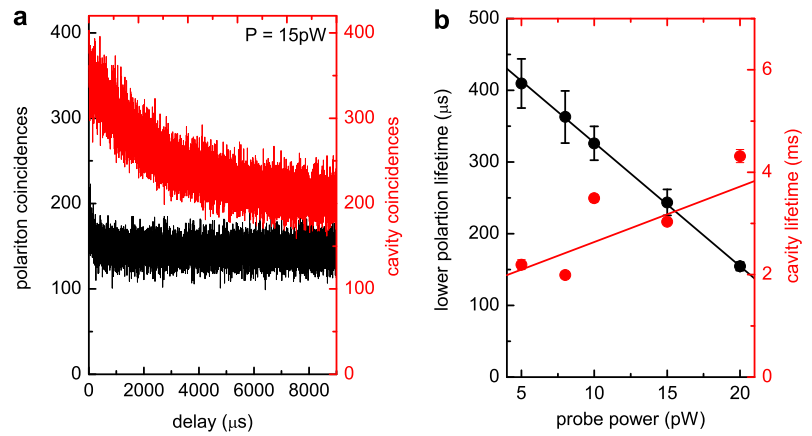

FIG. S 5: Lifetimes of the neutral quantum dot ground state and the charged/dark states. a) Auto-correlation traces on the lower polariton resonance (black trace) and the uncoupled cavity resonance (red trace) at a probe power of $15 \mathrm{pW}$. The lifetime ratio reveals that the system spends an order of magnitude more time in a state other than the neutral ground state due to laser-induced charge/spin pumping. b) Lifetimes of the neutral ground state (black trace) and the charged/dark states (red trace) as a function of excitation power.

\section{Determination of second order correlations at zero time delay}

The second order auto-correlation function $g^{(2)}(\tau)$ provides a direct measure of the statistics of emitted light. The degree of antibunching or bunching is simply given by the value at zero time delay $g^{(2)}(0)$. When the 
cavity is resonant with the exciton, we expect $g^{(2)}(0)=$ 0.34 on the lower polariton according to a simulation using our experimental parameters. The width of the dip / peak in $g^{(2)}(\tau)$ is given by the polariton state lifetime, which is in our case on the order of tens of ps. Since the APDs we use have a much higher timing jitter, they cannot be used to measure $g^{(2)}(0)$ under cw laser excitation. In order to overcome this limitation, we measure the auto-correlation function by exciting the system with short resonant pulses with a pulse-width similar to the polariton lifetimes. We define $g_{\text {pulsed }}^{(2)}(0)$ as the area of the central peak divided by the average area of peaks at other times. In order to have a realistic comparison between theory and our experimental data, we use a Monte Carlo wave function (MCWF) approach to obtain an estimate of $g_{\text {pulsed }}^{(2)}(0)$.

\section{A theoretical estimate of $g_{\text {pulsed }}^{(2)}(0)$}

The $g_{\text {pulsed }}^{(2)}(0)$ we measure is given by

$$
g_{\text {pulsed }}^{(2)}(0)=\frac{2 \int_{-T_{\text {rep }} / 2}^{T_{\text {rep }} / 2} d t_{1} \int_{t_{1}}^{T_{\text {rep }} / 2} d t_{2} G^{(2)}\left(t_{1}, t_{2}\right)}{\int_{-T_{\text {rep }} / 2}^{T_{\text {rep }} / 2} d t_{1} \int_{-T_{\text {rep }} / 2}^{T_{\text {rep }} / 2} d t_{2} I\left(t_{1}\right) I\left(t_{2}\right)},
$$

where $G^{(2)}\left(t_{1}, t_{2}\right)=\left\langle\mathrm{g}\left|\hat{C}^{\dagger}\left(t_{1}\right) \hat{C}^{\dagger}\left(t_{2}\right) \hat{C}\left(t_{2}\right) \hat{C}\left(t_{1}\right)\right| \mathrm{g}\right\rangle$, $I(t)=\left\langle\mathrm{g}\left|\hat{C}^{\dagger}(t) \hat{C}(t)\right| \mathrm{g}\right\rangle$, and $T_{\text {rep }}$ is the time difference between two laser pulses. Here, $|\mathrm{g}\rangle$ denotes the system ground state, before a laser pulse has excited the system. The system collapse operator $\hat{C}(t)=\sqrt{\kappa} \hat{a}(t)$ evolves according to the non-Hermitian effective Hamiltonian (in the interaction picture with the rotating frame of the laser center frequency) $H_{\text {eff }}(t)=H_{\mathrm{JC}}+H_{\text {int }}(t)-$ $\frac{i \hbar}{2}\left(\kappa \hat{a}^{\dagger} \hat{a}+\gamma \hat{\sigma}_{+} \hat{\sigma}_{-}+\gamma_{\mathrm{deph}}\right)$, where $\hat{a}$ is the cavity photon annihilation operator and $\hat{\sigma}_{+}, \hat{\sigma}_{-}$are the exciton creation and annihilation operators. $H_{\mathrm{JC}}$ is the JaynesCummings Hamiltonian and $H_{\text {int }}=\frac{\hbar \Omega(t)}{2}\left(\hat{a}+\hat{a}^{\dagger}\right)$ denotes the interaction with the Gaussian laser pulse, $\Omega(t)=\Omega_{0} \exp \left(-2 \ln (2) t^{2} / T_{\text {pulse }}^{2}\right) \cdot \kappa$ denotes the cavity dissipation rate, $\gamma$ the exciton spontaneous recombination rate and $\gamma_{\text {deph }}$ the exciton pure dephasing rate; the latter may stem from phonon mediated coupling of the two dressed polariton states. In order to determine $G^{(2)}\left(t_{1}, t_{2}\right)$ and $I(t)$, we use a Monte Carlo wavefunction approach, as described in Ref. [5. The calculations are performed with the experimentally determined values, $\lambda_{\text {exciton }}=937.25 \mathrm{~nm}, \hbar g=141 \mu \mathrm{eV}, \hbar \kappa=53 \mu \mathrm{eV}$, $\hbar \gamma=0.66 \mu \mathrm{eV}$, and $\hbar \gamma_{\mathrm{deph}}=13 \mu \mathrm{eV}$. For a typical pulse duration of $72 \mathrm{ps}$ (FWHM), a pulse energy of $82 \mathrm{eV}$ and an estimated laser - cavity coupling efficiency of $2 \%$, we obtain $\hbar \Omega_{0} \approx 24 \mu \mathrm{eV}$.
In the experiments, the suppression of the noninteracting reflected laser light is not perfect $(\approx 1$ : 50 000), and consequently, our measurements are subject to interference effects between cavity emission and laser light. Possible reasons for incomplete suppression are a mixing of polarization states when the light traverses the collection fiber or the analyzer, or depolarization effects due to the reflection at the photonic crystal membrane. We take the phase difference $\phi$ between the laser light that drives the Jaynes-Cummings system and the non-interacting (reflected) laser to be $\phi=-\pi / 2$; this value corresponds to the (experimentally optimized) largest ratio between detected cavity photons and laser background. We emphasize that we have a control over both the amount of laser suppression and the phase difference $\phi$, by the use of fiber-polarization controllers.

As described in the previous section, the system is in the desired neutral ground state in only $r=30-40 \%$ of the cases in average. Experimentally, we cannot distinguish between on- and off-resonant cases, and the resulting correlation function is a sum of both on-resonant and the Poissonian off-resonant correlation functions. Thus, at zero time delay

$$
\tilde{g}_{\text {pulsed }}^{(2)}(0)=\frac{r E_{\mathrm{on}}^{2} g_{\mathrm{pulsed}}^{(2)}(0)+(1-r) E_{\mathrm{off}}^{2}}{r E_{\mathrm{on}}^{2}+(1-r) E_{\mathrm{off}}^{2}},
$$

where $E_{\text {on }}$ is the average detected energy per pulse of the Jaynes-Cummings system, as described above, and $E_{\text {off }}$ the respective pulse energy of the uncoupled cavity emission, together with the non-interacting laser light background. Close to the cavity-exciton resonance, $g_{\text {pulsed }}^{(2)}(0)$ should exhibit a bunching peak on the exciton transition (see Fig. S 6a). This bunching-feature is absent in $\tilde{g}_{\text {pulsed }}^{(2)}(0)$ (Fig. S $6 \mathrm{~b}$ ) due to the predominant detection of photons scattered from the uncoupled cavity mode. For the rest of the discussion here and in the main text, we will omit the tilde and refer to equation (2) as the definition of correlations at zero time delay.

\section{Pulse parameters}

Simulations on the lower polariton reveal a reduction of the non-classical part $\left|g_{\text {pulsed }}^{(2)}(0)-1\right|$. The reasons are repeated photon absorption/emission events within a single laser pulse, excitations of more than one resonance due to the broad pulse spectrum and the large lasercavity coupling with $\Omega_{0} \lesssim \kappa$. For Fourier-limited Gaussian laser pulses, we find a minimum of $g_{\text {pulsed }}^{(2)}(0)=0.75$ for a laser pulse duration of about $50 \mathrm{ps}$, as shown in Fig. S 7a. In the experiments, we measure the spectrally filtered pulses to have slightly larger durations of $T_{\text {pulse }} \approx 72 \mathrm{ps}$. This corresponds to a ratio between pulse duration and polariton lifetime of $\frac{\kappa}{2} \cdot T_{\text {pulse }} \approx 2.9$. For in- 

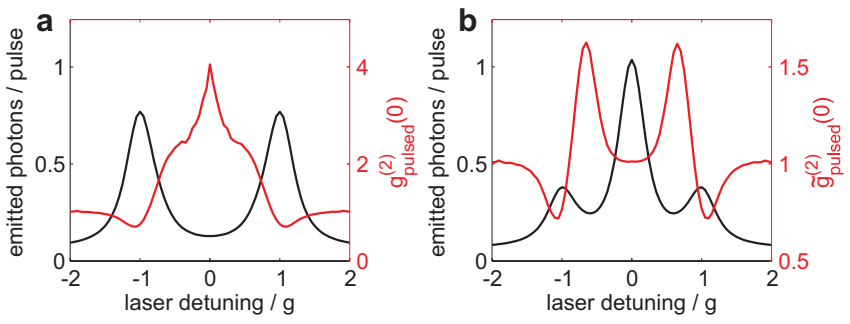

FIG. S 6: MCWF simulations on cavity-exciton resonance. a) Assuming that there is no QD-blinking, the system spectrum exhibits only the two polariton peaks (black line). There is a strong bunching peak for zero laser-exciton detuning in $g_{\text {pulsed }}^{(2)}(0)$ (red line). b) Simulations of the real system with QD-blinking $(r=0.4)$ yield a spectrum (in time average) with a three-peak feature (black line); the middle uncoupled cavity peak causes the bunching feature in $\tilde{g}_{\text {pulsed }}^{(2)}(0)$ to vanish (red line).
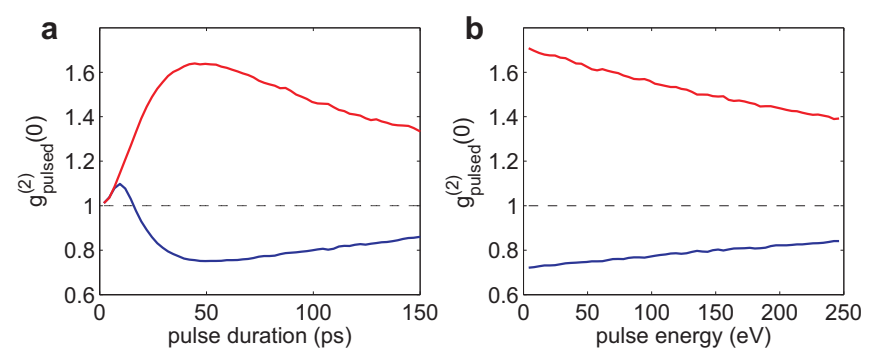

FIG. S 7: Simulations of $g_{\text {pulsed }}^{(2)}(0)$ for different pulse parameters. Blue traces indicate probing of the lower polariton and red traces the lower two-photon resonance. a) $g_{\text {pulsed }}^{(2)}(0)$ as a function of pulse duration for a fixed pulse energy of $82 \mathrm{eV}$. b) $g_{\text {pulsed }}^{(2)}(0)$ as a function of pulse energy for 72 ps pulses. creasing pulse energy, the calculated $\left|g_{\text {pulsed }}^{(2)}(0)-1\right|$ decreases, as shown in Fig. S 7b. We typically work with pulse energies between 40 and $100 \mathrm{eV}$, which corresponds to $0.6-1.5$ scattered photons per pulse on laser-cavity resonance for the estimated coupling efficiency of $2 \%$.

The efficiency of the generation of non-classical light improves with a slight cavity-exciton detuning. A blue detuning of the cavity by $g$ with respect to the exciton resonance yields $g_{\text {pulsed }}^{(2)}(0)=0.63$ for the lower polariton. This reduction in $g_{\text {pulsed }}^{(2)}(0)$ originates predominantly from the prolongation of the polariton lifetime which reduces the likelihood of multiple photon absorption events within a single pulse.

[1] When comparing polariton and cavity lifetimes in Fig. $\mathrm{S} 5 \mathrm{~b}$, we would expect efficient emission from the uncoupled cavity already at $5 \mathrm{pW}$. However, cavity emission is strongly suppressed, since an initial polariton excitation is required to bring the QD into a charged/dark state.

[2] Faraon, A. Locally controlled photonic crystal devices with coupled quantum dots: physics and applications. PhD Thesis, Stanford University, (2009).

[3] Cassabois, G. personal communication, (2011).

[4] Faraon, A. et al. Coherent generation of non-classical light on a chip via photon-induced tunneling and blockade. $\mathrm{Na}$ ture Physics 4, 859-863 (2008).

[5] Molmer, K. et al. Monte Carlo wave-function method in quantum optics. J. Opt. Soc. Am. B 10, 524 (1993). 